\title{
Nitrate fluorimetric analysis using an active optical fiber
}

\author{
B. Mahieuxe ${ }^{1}$, M.-C. Carré ${ }^{1}$, J.-C. André ${ }^{1}$, A. Revillon ${ }^{2}$ and M.-L. Viriot ${ }^{1, *}$ \\ ${ }^{1}$ DCPR-GRAPP, UMR 7630 CNRS-INPL, Groupe ENSIC, 1 rue Grandville, 54000 Nancy, France. \\ ${ }^{2}$ LMOPS, CNRS Lyon-Solaize, BP. 24, 69390 Vernaison, France.
}

\begin{abstract}
With the objective to use a fluorimetric determination of nitrate based on fluorescein as the sensitive fluorescent indicator, an active optical fiber was elaborated and its response was tested using a specifically adapted fluorimeter. So, the three required chemical steps to fix fluorescein via a covalent bond on the silica fiber tip will be described, as well as the amount of grafted probe and the stability of the optrode in sulfuric acid (the required medium for the analysis). Then, for the determination of nitrate, the active optrodes were connected to the specially designed optical fiber fluorimeter. The integrating optical fiber sensor response is linear for concentrations between $210^{-6}$ and $10^{-5} \mathrm{~mol} \mathrm{NO}_{3}^{-} \mathrm{L}^{-1}$ (i.e. between 0.12 and $0.62 \mathrm{mg} \mathrm{NO}_{3}^{-} \mathrm{L}^{-1}$ in the reactive medium). One optrode has to be used for each analysis (or each set of analyses if all the grafted fluorescein is not consumed).
\end{abstract}

Key words. nitrate - fluorescence - fluorescein - silica grafting.

These last past years, nitrate ions have been in vogue pollutants because often incriminated in aquatic pollutions. Very currently used as chemical fertilizers, nitrate can be transformed, when ingested, into carcinogenic nitrosamines in the human body. So it is necessary to monitor the concentrations of these anions, even as traces, particularly in drinking waters or in order to prevent pollution accidents in environmental waters. We recall that the recommanded nitrate concentration by the European Community for drinking water is less than $50 \mathrm{mg} \mathrm{NO}_{3}^{-} \mathrm{L}^{-1}$.

Besides several methods (ion chromatography, absorption spectrometry, etc. [1,2]), fluorimetry is generally described as a very sensitive, selective, simple and quick technique (see reference 3 for a review). Moreover, use of an optical fiber would allow both remote excitation and fluorescence emission guiding. Then, our objective was to develop a sensor based on fluorescence measurements as a system currently able to rapidly measure, in situ and specifically, low nitrate rates.

Following a large bibliographical study [3], a fluorimetric method for the determination of nitrate was selected and particularly the one using fluorescein as a fluorescent indicator [4,5]. Fluorescein advantages are its non toxicity and the fact that it has several commercially available derivatives, making easier its covalent immobilization on an optical fiber. The method consists in the determination of nitrate ions in concentrated sulfuric acid, by measuring their quenching effect on the fluorescence of fluorescein, owing to the formation of a non fluorescent dinitrated compound, as indicated in figure 1 . The analysis of nitrate ions in homogeneous acidic medium was largely studied $[4,5]$ and we reproduced the method with our own equipment. Even the acidic medium, the study of the fluorescence emission is still

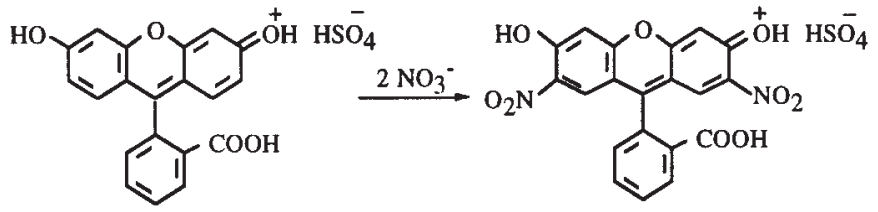

Fig. 1. Reaction between the nitrate ions and fluorescein, in concentrated sulfuric acid.

possible, of course the fluorescence characteristics have changed: lower quantum yield and blue shift of the emission [6].

With the idea of the development of an active optrode, the major drawback is the requirement of concentrated sulfuric acid, $89 \%$ minimum, which might be harmful for its stability. Therefore, to obtain a more stable optrode regarding the immobilization of fluorescein, it would be preferable to create a covalent bond. The three required steps for a covalent immobilization of fluorescein on the silica tip of the optical fiber are summarized in figure 2. Such a fixation would prevent or at least limit the desorption or wash out phenomena which would happen for the optrode using in a highly aggressive media, such as $95 \%$ sulfuric acid. Two minor disadvantages are respectively the too long reaction time (i.e. $45 \mathrm{~min}$; in fact we will show that this time might be reduced to $30 \mathrm{~min}$ ) and the irreversibility of the reaction which will limit the method to an integrating sensor. 


\section{Original articles}
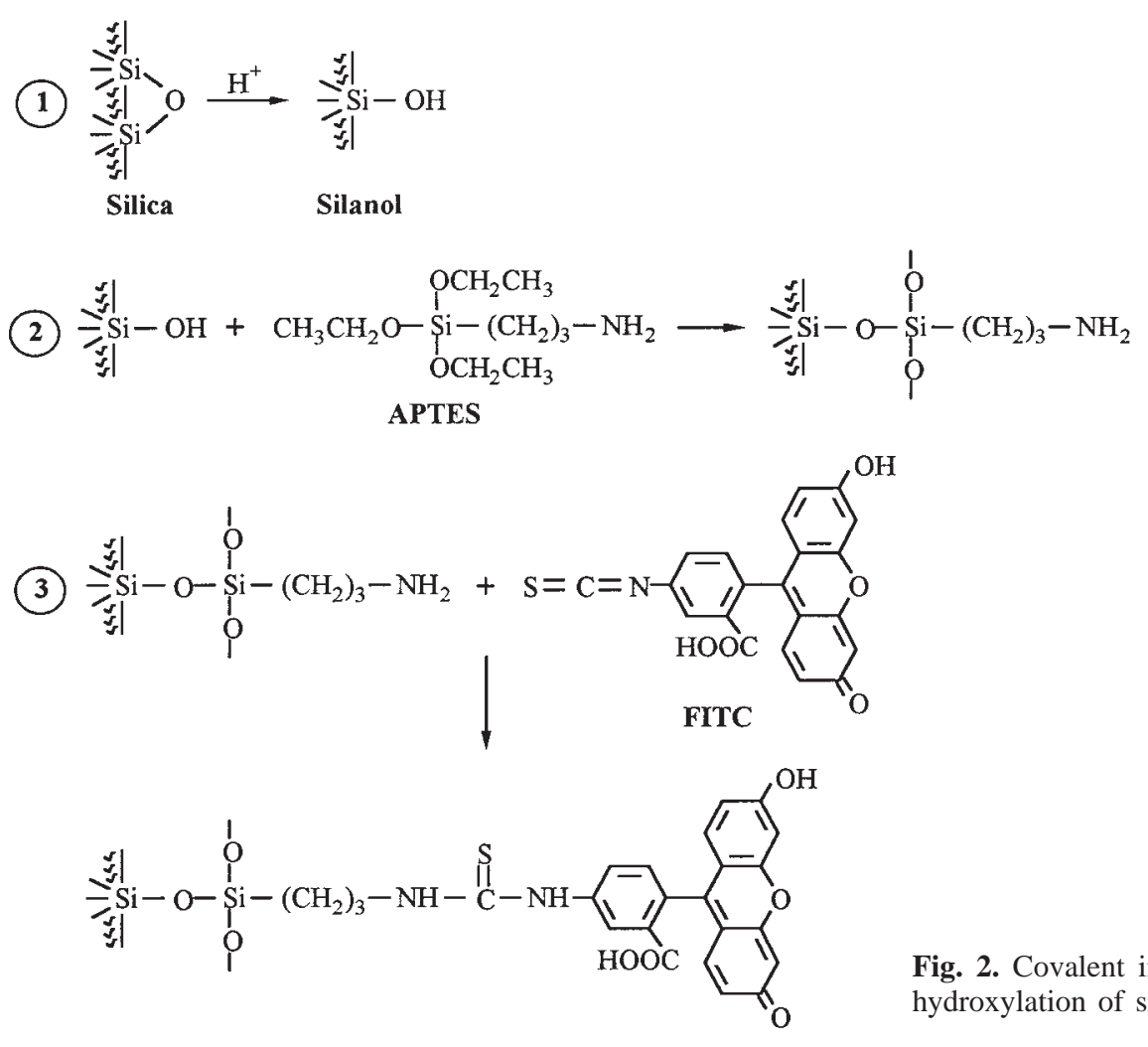

Fig. 2. Covalent immobilization of fluorescein in three steps. (1) hydroxylation of silica; (2) silanization; (ㅁ) fluorescein grafting.

\section{Experimental section}

\section{Chemical elaboration of the active optrode}

\section{Reagents}

Fluorescein isothiocyanate (FITC, isomer I), 3-aminopropyltriethoxysilane (APTES, $98 \%$ ), potassium nitrate (puriss > $99 \%$ ) and 95-97 \% sulfuric acid (puriss) were purchased from Fluka (F-St Quentin Fallavier). APTES was distilled under vacuum and stored under inert atmosphere. Crystallized sodium tetraborate (borax, for analyses and buffers) was a Merck product (D-Darmstadt). Toluene (for anhydrous analyses from sds, F-Peypin, with a water content lower than $0.0075 \%$ ) was preserved on $4 \AA$ molecular sieves. $69 \%$ minimum nitric acid (R.P. Normapur ${ }^{\mathrm{TM}}$ ) was purchased from Prolabo (F-Paris). The water used was demineralized. For the $\mathrm{pH} 8.6$ borate buffer solution, the preparation was as follows: $250 \mathrm{~mL}$ of a $0.025 \mathrm{M}$ borax solution and $67.5 \mathrm{~mL}$ of a $0.1 \mathrm{~N}$ hydrochloric acid solution were introduced in a $500 \mathrm{~mL}$ flask. The mixture was then completed to $500 \mathrm{~mL}$ with demineralized water [7]. The potassium nitrate was dried at $100{ }^{\circ} \mathrm{C}$ before using. Three exactly about $10^{-4}, 10^{-3}$ and $10^{-2} \mathrm{~mol} \mathrm{NO}_{3}^{-} \mathrm{L}^{-1}$ aqueous solutions of nitrate were prepared.

\section{Optical fiber}

The optical fiber used was a multimode step index one, "silica-silicon" type, doped with hydroxyl ions (about 300 ppm)

purchased from Foretec (F-Montbrison). The core was in ultra pure synthetic silica glass, the optical cladding was in silicon, the protection coating was a ethylene-polytetrafluoroethylene ETFE ("tefzel") copolymer. The core diameter was $1 \mathrm{~mm}$, the numerical aperture was 0.4 . The stripper used for silicon sheath optical fiber was commercially available as O.F. Stripper "S" (Lumer S.A., F-Bagnolet) and contained methylene chloride, sulfuric acid $(0.5 \%)$ and detergents.

\section{Reactor}

In order to run the chemical steps required for the FITC grafting on the stripped fiber tip, without damaging the silicon optical cladding of the sheathed part, it has been necessary to develop a suitable reactor (Fig. 3).

This reactor, in whole pyrex, consisted of two parts: (A) a part composed of 4 identical tubes, which inside diameter was a few higher than that of the optical fiber including its protection coating. The inner diameter of the lower extremity of each tube was smaller, so as to let only the optical fiber stripped part overlapping (just higher than $1 \mathrm{~mm}$ ), and (B) a lower part, on which the former part well fitted and in which the grafting reactions occurred. It included an inlet for reagents and solvents introduction, an inlet for nitrogen injection and a rod neck to set a reflux condenser.

The optical fibers were introduced into the tubes, their stripped end dipping in the reactive medium which was flushed with the edge of each tube. The higher part 


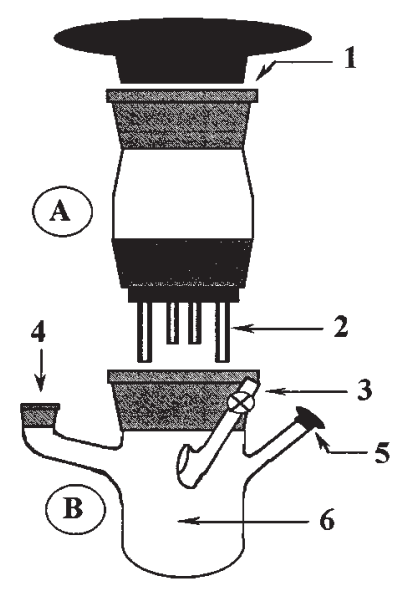

Fig. 3. Reactor design for the fluorophore grafting on the tip of an optical fiber. (A) higher part: (1) fibers introduction, (2) capillary tubes; (B) lower part: (3) nitrogen inlet; (4) reflux condenser position; (5) reagents introduction; (6) reactive zone.

containing optical fibers must be closed in order to prevent the rise of the reactive medium into the tubes by capillarity.

All the glass-ware used was dried at $120{ }^{\circ} \mathrm{C}$ for the silanization reaction.

\section{Optical fiber preparation}

Optical fiber strands, $12 \mathrm{~cm}$ length, were stripped at one of their ends, on about three centimeters in length. The ETFE protection sheath was taken off with a cutter. The silicon optical cladding was destroyed by plunging the tip of the fibers in the stripper solution. The fibers were then plentifully rinsed with water and acetone.

\section{Hydroxylation}

The creation of silanols (Fig. 2, step 1 ) was obtained by dipping the stripped tips of the fibers into a $1.5 \mathrm{~N}$ nitric acid solution, at $50{ }^{\circ} \mathrm{C}$ for one hour. The strands were then plentifully rinsed with water and acetone and dried at $120{ }^{\circ} \mathrm{C}$ in an oven.

\section{Silanization}

The silanization reaction (Fig. 2, step 2) was conducted under dried nitrogen (bubbling in sulfuric acid and then passing through a column filled with phosphoric anhydride). The hydroxylated fibers were introduced in the reactor containing a $10 \%(\mathrm{v} / \mathrm{v})$ APTES solution in toluene. The reaction was led under stirring at $110{ }^{\circ} \mathrm{C}$ for 5 hours. The fibers were then rinsed in the reactor and under nitrogen, three times with anhydrous toluene and three times with pure acetone, then left in pure acetone under stirring and in nitrogen atmosphere for 2 days and lastly rinsed with acetone. They were then dried at $100{ }^{\circ} \mathrm{C}$ in an oven.

\section{Fluorescein grafting}

In order to perform the covalent bonding of fluorescein (Fig. 2, step $\underline{3}$ ), the silanized fibers tips were immersed in a $\mathrm{pH} 8.6$ borate buffer solution which was FITC saturated (about $510^{-3} \mathrm{~mol} \mathrm{~L}^{-1}$ ). The reaction occurred at $35^{\circ} \mathrm{C}$ during 5 days [8]. Then fibers were rinsed several times with acetone and water, until no FITC fluorescence was detected in the washes. The fibers were then dipped in demineralized water at $30{ }^{\circ} \mathrm{C}$ for 2 days in order to wash out unlinked FITC traces. They were finally once again rinsed several times with water, dried and stored in a desiccator until their use. The fluorescence spectra of fluorescein in solution and grafted are already published [9].

\section{Optical device and optrode set-up}

The adapted fluorimeter was designed by Gaucher et al. [10] (Fig. 4). It includes: (1) a Coherent ${ }^{\circledR}$ ionized argon laser $\left(\lambda_{\mathrm{em}}=457.9 \mathrm{~nm}\right)$, Innova 90-4 model, $(\mathbf{2})$ a prism guiding the excitation light towards the optical fiber, (3) a photodiode for the correction of the possible fluctuations of the laser emission (reference beam), (4) a lens to focus the excitation beam on the tip of the optical fiber, (5) a "pin hole" (macroscopic hole with a diameter of $1 \mathrm{~mm}$ ) to spacially filter the laser and to eliminate the halo of parasite light around the beam, (6) a parabolic holed mirror (hole diameter of $3 \mathrm{~mm}$ ), which was got through by the excitation beam and allowed the collection of maximum fluorescence on the tip of the fiber and focusing it to the detection system, (7) an interference filter to eliminate light with wavelengths below to $500 \mathrm{~nm}$ (elimination of parasite laser reflections and of a part of fluorescence, optimum of which was found at about $485 \mathrm{~nm}),(8)$ a lens to focus the fluorescence to the photomultiplier, (9) a photomultiplier, its supplying and electronics (10), for the signal treatment. In spite of the cares, the laser light can be diffused, particularly by the prism and can reach the photomultiplier. Black screens (11) were then placed in order to dissociate the excitation part (laser) from the emission one (fluorescence) of the set-up. A silica-silicon optical fiber (12), of $1 \mathrm{~mm}$ diameter and $2 \mathrm{~m}$ length, connected the fluorimeter to the optrode. It was protected on all its length by a thermoretractable sheath to discard all parasite light or fluorescence phenomena. The end of this fiber, where occurred the measurement, was fitted out with a SMA connector to support the optrode (13). To fix, in a reproducible way, the active optrodes on the tip of the fluorimeter optical fiber, SMA connectors were used. For that, connectic kits commercialized by Radiall ${ }^{\circledR}$ (F-Rosny sous Bois) were employed. The connectors used were F.SMA5 type and were theoretically designed for optical fibers with core diameter between 100 and $140 \mu \mathrm{m}$. The inner diameter of the connector tube has been then enlarged to make it to accept fibers with core diameter of $1 \mathrm{~mm}$.

\section{Measurement conditions and procedure}

As the surface coverage of each optrode might not generally have exactly the same value, one optrode and only one was used for each set of measurements. Indeed, successive 


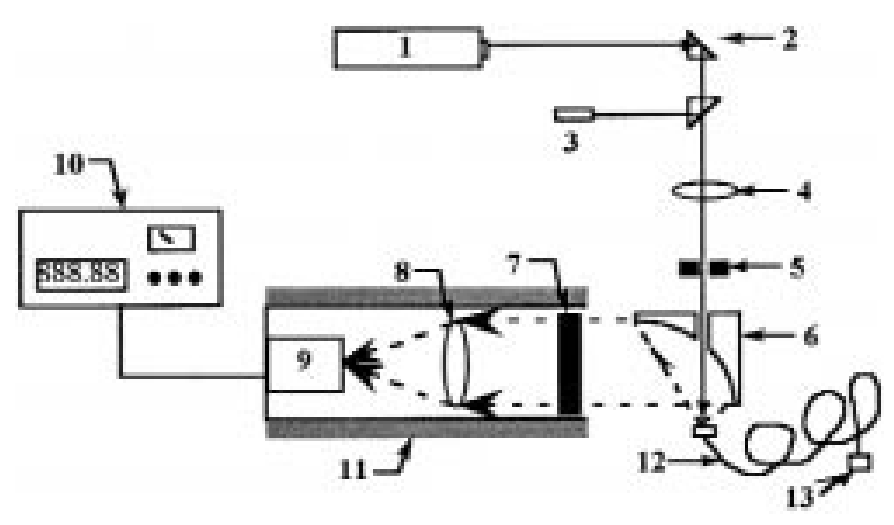

Fig. 4. Optical fiber fluorimeter. Solid direction line: laser excitation; dotted direction line: optrode fluorescence.

adds of nitrate were analyzed and the optrode cumulated the different responses because of its non reversibility (integrating sensor). For each fluorescence measurement, a reference test was realized: an optical fiber, with one of its extremities stripped but with no grafting of fluorescein, was linked to the fluorimeter fiber. So the background of the medium was measured and taken off from the fluorescence signals. When the optrode was replaced by a fiber without grafting and vice versa, the part which had been in contact with the sulfuric acid was carefully washed with demineralized water, then with acetone and dried. A measurement of the fluorescence before each new add of nitrate was achieved too in order to count all damages of the grafted layer which was inevitably caused by the sulfuric acid at the time of the last measurement. The optrode grafted part was fully dipped in a known volume of sulfuric acid $(10 \mathrm{~mL})$. The medium was always under strong stirring. The aqueous sample of nitrate was injected with the help of a syringe in the bottom of the flask containing the sulfuric acid to allow a better homogeneity. The entire injected volume must not go beyond $1 \%$ (v/v) of the initial volume of the sulfuric acid $(100 \mu \mathrm{L}$ in totality). The sulfuric acid was not renewed between the measurements, in order to keep the balance established between the optrode and the medium. As the time for the chemical reaction between nitrate ions and fluorescein was between 30 and $45 \mathrm{~min}$, the laser beam was opened only for the fluorescence analysis in order to minimize at the very most the photodegradation of fluorescein. In addition, a study of the influence of the power laser on the photodegradation was performed (see further).

\section{Results and discussion}

\section{Covalent immobilization of fluorescein on the optical fiber}

The silica core of the optical fiber was at first activated in acidic medium, to create the maximum reactive silanols sites on its surface. This step was very important to obtain a high level of fluorescein surface concentration. The following silanization reaction governs the final grafting quality. The process was a condensation reaction between the surface silanols and the siloxane part of APTES, with the concomitant elimination of APTES ethoxy groups (one or two, but never three [11]). When it occurred with water traces, it ensued the hydrolysis of the silane leading to the uncontrolled formation of a polysiloxane network [12]. Indeed, dior trifunctional silanes led to a very easily hydrolysis giving rise to the formation of a linear or a branch polymer respectively. The presence of water traces in the solvent and/or the reagents and/or adsorbed on the glass-ware and/or the optical fiber sides, as well as ambient moisture, were sufficient to hydrolyze the 3-aminopropyltriethoxysilane into a poly[(aminopropyl)siloxane] network. If this polysiloxane amplified 100 times the number of reactive sites towards FITC, it also showed several drawbacks: (i) the main one was the fact that the diffusion coefficients of the solutes were much lower in polymer grafts than in monolayer ones; experimentally, this corresponded to a longer reaction time between bonded fluorescein and nitrate, which initially was $45 \mathrm{~min}$, but also to a loss of the sensor sensitivity towards nitrate ions, (ii) the polysiloxane chains were preferably formed in the solution and laid down on the surface (entanglement of chains) and they were practically not or little covalently bound to the silica, (iii) the control of the polymerization reaction was very difficult, giving often a non reproducible grafting, instead of a monomolecular layer, and (iv) at last, the polysiloxane network, by raising a lot the surface coverage, could contribute to draw closer the immobilized fluorescein molecules and promote the formation of non fluorescent dimers [13].

So, in order to have the more coherent and the more reproducible results as possible, a monomolecular grafting was attempted, by working in severe anhydrous conditions. The fluorescein immobilization was achieved via its isothiocyanate derivative. The condensation reaction between the isothiocyanato function and the APTES amino group was conducted in a low alkaline medium, leading to the formation of a thiourea bond (Fig. 2, step $\underline{3}$ ).

The amount of bound fluorescein on the silica fibers was determined by treating these latters with a $0.1 \mathrm{~N}$ sodium hydroxyde solution. The fluorescence of the alkaline solution was next measured by comparison to a calibration curve. According to the method used, the covalently surface coverages obtained for this optrodes study were included between 2 and $3 \mu \mathrm{mol} \mathrm{m} \mathrm{m}^{-2}$. In theory, the maximal surface concentration of the FITC as a monomolecular layer is of the order of $2.5 \mu \mathrm{mol} \mathrm{m}{ }^{-2}$ [9].

The reproducibility for the amount of grafted fluorescein is roughly in the range of $30 \%$. This led us to use the same optrode for a series of experiments.

\section{Validation of the fluorimeter}

In order to get a reliable analysis with the optical fiber fluorimeter, as described in figure 4, first we run the 


\section{Original articles}

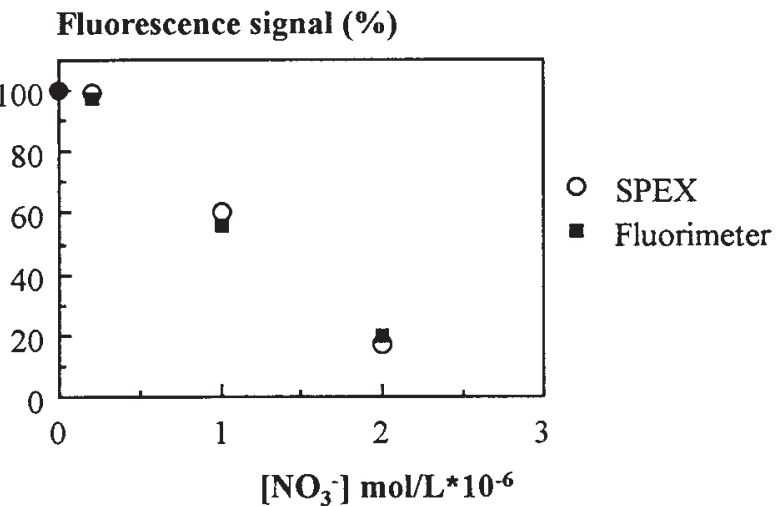

Fig. 5. Comparison of the fluorescence response for the analysis of nitrate ions in an homogeneous dosage: fluorescence measurement in a cuvette with the spectrofluorimeter SPEX and through the passive optical fiber of the designed fluorimeter.

determination of nitrate in the homogeneous way using the fluorimeter with a passive optrode (the $2 \mathrm{~m}$ length optical fiber of the fluorimeter was stripped of $2 \mathrm{~cm}$ ). In parallel, the analysis of the same samples was performed by using a conventional spectrofluorimeter (SPEX fluorolog II). The results relative to the two methods (Fig. 5) indicate a good correlation and put trust in the optical fiber fluorimeter.

\section{Laser power to get the best compromise between sensitivity and photostability for the grafted fluorescent tracer}

It is well known that fluorescein might be photodegraded under light exposition, even its low disappearance quantum yield [6]. So, we have studied the photodegradation of FITC immobilized on the optical fiber, especially by changing the laser power (going from 22 to $6 \mathrm{~mW}$ ). Of course, the laser power will have an influence on the intensity of the fluorescence signal (in theory, the highest signal will correspond to the highest power). To perform the experiment, the optrode was immersed in concentrated sulfuric acid without stirring and the variation of the fluorescence signal was analyzed over time by guiding the laser beam at different powers in a continuous way into the fiber. For a $22 \mathrm{~mW}$ power, photodegradation of fluorescein is observed with a rapid decrease at the starting and further a slower degradation rate (Fig. 6). For a $11 \mathrm{~mW}$ power, the response is roughly stable at the beginning with a very gentle decrease progressively with time. For a still lower power $(6 \mathrm{~mW})$, the fluorescence signal is stable with time. Then, to get a good compromise between sensitivity and photodegradation, a laser power comprised between 5 and $10 \mathrm{~mW}$ was generally chosen for nitrate determination.

\section{Optrode stability}

Before to start the determination of nitrate ions with the active optrodes, it was necessary to know their behaviour
Fluorescence signal (\%)

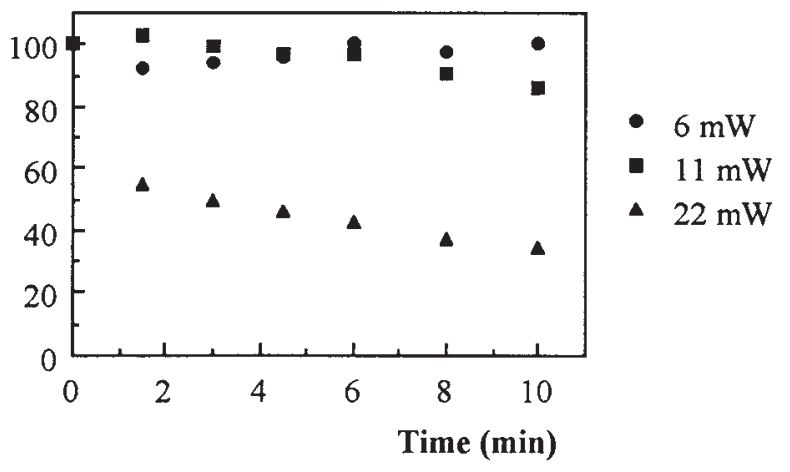

Fig. 6. Influence of the laser power on the fluorescence signal over time of exposition (relative fluorescence intensity value to the value at time $t=0$, for each laser power value).

under the measuring conditions over a period of several hours. In a first set of experiments [9], the grafting stability was tested by introducing one optrode in a known volume of $95 \%$ sulfuric acid under vigorous stirring and measuring the fluorescence of the solution at 15 min intervals by comparison to a calibration curve (sulfuric acid was renewed after each measurement). After 45 min (time usually required for the nitration of fluorescein), the amount of fluorescein loss was scarcely higher than $2 \%$ of the overall binding, and after 3 hours, it did not reach $10 \%$. To meet the measuring conditions, in a second set of experiments, the grafted area of an optrode was also plunged in sulfuric acid under strong stirring, but without change of sulfuric acid between the measurements. The fluorescence signal was noted at regular intervals of time (Fig. 7). The signal rapidly decreased the first $45 \mathrm{~min}$, next leaned towards stabilization: a diffusive equilibrium of the fluorescein was probably established between the grafted layer and the sulfuric acid medium, which braked the damage of the optrode in time. After $135 \mathrm{~min}$, it remained roughly $80 \%$ of the initial signal, which was very acceptable for a prolonged use of the optrode.

\section{Application of the active optrodes for the nitrate determination}

In order to know the sensitivity of the achieved optrodes, their response has been studied for various ranges of nitrate concentrations (concentrations in the reactive medium, after their dilution by sulfuric acid; the dilution factor varied from 100 to 500 according to the sample volume added).

\section{Nitrate concentrations in the range $210^{-7}$ to $10^{-6} \mathrm{~mol} \mathrm{NO}_{3}^{-} \mathrm{L}^{-1}\left(0.012\right.$ to $\left.0.062 \mathrm{mg} \mathrm{NO}_{3}^{-} L^{-1}\right)$}

These contents corresponded to the range of concentrations used in the original homogeneous fluorimetric method $[4,5]$. The reaction time was of $45 \mathrm{~min}$. The optrodes did not seem 


\section{Original articles}

Fluorescence signal (\%)

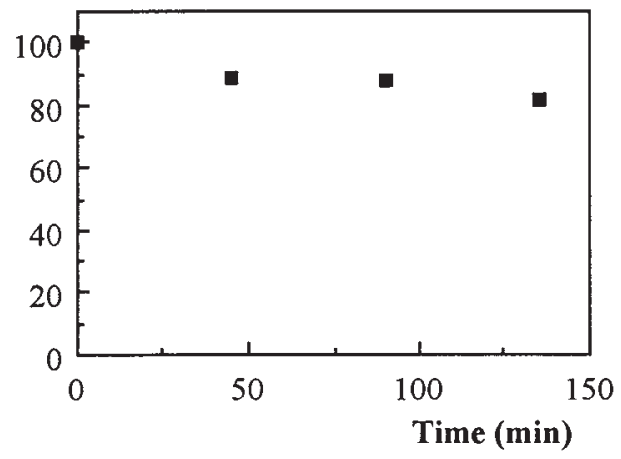

Fig. 7. Optrode stability in sulfuric acid: variation of the fluorescence signal over time.

to be sensitive towards nitrate in this range of concentrations (Fig. 8) and the low signal variations observed were essentially due to the altering of the coverage by sulfuric acid.

Nitrate concentrations in the range $210^{-6}$ to $10^{-5} \mathrm{~mol} \mathrm{NO}_{3}^{-} \mathrm{L}^{-1}$ (0.12 to $0.62 \mathrm{mg} \mathrm{NO}_{3}^{-} \mathrm{L}^{-1}$ )

For a ten times higher concentrations as the previous ones and a reaction time of $30 \mathrm{~min}$, the optrode response was linear (Fig. 9). This very promising result proved the feasibility of the integrating optical fiber sensor developed for the nitrate determination in the concentrations range $210^{-6}$ to $10^{-5} \mathrm{~mol} \mathrm{NO}_{3}{ }^{-} \mathrm{L}^{-1}$ ( 0.12 to $\left.0.62 \mathrm{mg} \mathrm{NO}_{3}^{-} \mathrm{L}^{-1}\right)$, which corresponded to nitrate samples of initial concentrations roughly included between $210^{-4}$ and $210^{-2} \mathrm{~mol} \mathrm{NO}_{3}^{-} \mathrm{L}^{-1}$ (i.e. between 12 and $1200 \mathrm{mg} \mathrm{NO}_{3}^{-} \mathrm{L}^{-1}$ in the sample before its dilution in the sulfuric acid medium).

If the reduced reaction time of $30 \mathrm{~min}$ in comparison to the usual one of $45 \mathrm{~min}$ led to a lower sensitivity of the optrodes (decrease of the slope of the straight line; results not shown), it remained very acceptable for the analysis and mostly it allowed a quicker measurement.

Nitrate concentrations higher than $10^{-5} \mathrm{~mol} \mathrm{NO}_{3}^{-} \mathrm{L}^{-1}$ (higher than $0.62 \mathrm{mg} \mathrm{NO}_{3}^{-} \mathrm{L}^{-1}$ )

Although the nitrate determination for concentrations higher than $10^{-5} \mathrm{~mol} \mathrm{NO}{ }_{3}^{-} \mathrm{L}^{-1}$ could be uninteresting, we wanted to check how far the sensor response was linear. In fact, for concentration higher than $10^{-5} \mathrm{~mol} \mathrm{NO}_{3}{ }^{-} \mathrm{L}^{-1}$ the response is not anymore linear, it very quickly reached a minimum showing the saturation of the sensor: the bound fluorescein to the tip of the optical fiber was consumed at the most by the reaction with nitrate. This minimum, here situated around $40 \%$ of the initial signal, both depended on the reaction time and the surface coverage on the tip of the fiber.
Fluorescence signal (\%)

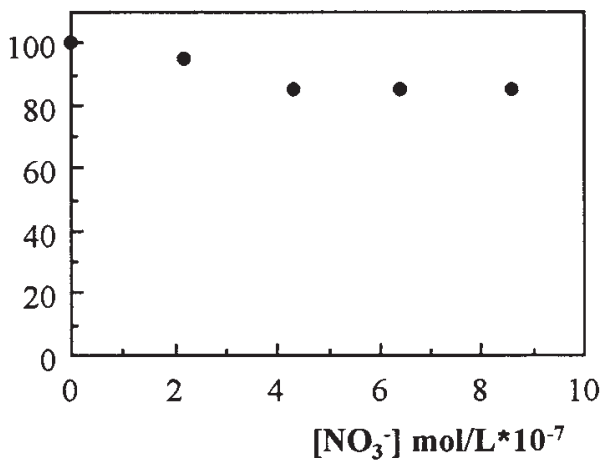

Fig. 8. Response of an optrode to nitrate concentrations between 2 $10^{-7}$ and $10^{-6} \mathrm{~mol} \mathrm{NO}_{3}^{-} \mathrm{L}^{-1}$.

\section{Fluorescence signal (\%)}

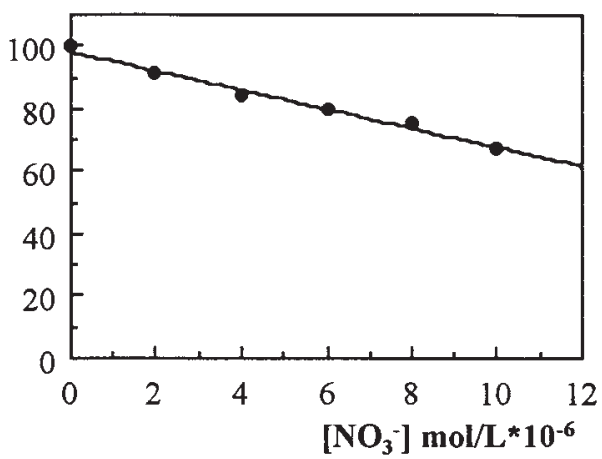

Fig. 9. Response of an optrode to nitrate concentrations between 2 $10^{-6}$ and $10^{-5} \mathrm{~mol} \mathrm{NO}_{3}^{-} \mathrm{L}^{-1}$.

Fluorescence signal (\%)

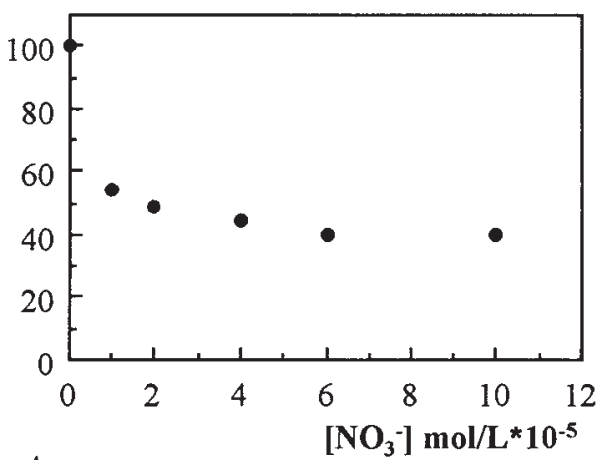

Fig. 10. Response of an optrode for nitrate concentrations higher than $10^{-5} \mathrm{~mol} \mathrm{NO}_{3}^{-} \mathrm{L}^{-1}$. 


\section{Conclusion}

The optical fiber sensor described here has been successfully tested for the nitrate ions determination. The results have proved a good enough stability of the optrodes in sulfuric acid, which make them able to a prolonged use. Thus, it was shown up the possible application of the active optrodes to the quantitative measurement of the nitrate ions in the concentrations range between 0.12 and $0.62 \mathrm{mg} \mathrm{NO}_{3}{ }^{-} \mathrm{L}^{-1}$ in the reactive medium. Then, the developed active optrodes have a sensitivity towards nitrate comparable, indeed higher, to that of the optical sensors already achieved for these anions [14].

However, the optrode sensitivity is about ten times lower than that of the original homogeneous fluorimetric method, this is due to a more difficult reaction of nitrate at the interface liquid (reactive medium) - solid (optical fiber) than in liquid phase.

The two important parameters to improve the sensor abilities are: (i) the reaction time which is already too long for a sensor and (ii) the surface concentration of the fluorescent probe on the optrodes, indeed the higher the surface coverage, the less sensitive the final sensor will be towards low nitrate concentrations (too small fluorescence diminution in comparison to the original signal). Nevertheless, the surface coverage must be sufficient in order to keep a measurable fluorescent signal; moreover, as the reaction is irreversible, the grafted fluorescent probe is consumed by the reaction. The surface coverage must also be sufficiently high in order to carry out several measurements with only one and same optrode (integrating sensor). Thus the aim is to modulate this last parameter, especially by perfectly governing the chemical methods of covalent immobilization and making a compromise between the sensitivity and the sensor measurement capability.

The elaborated optical fiber chemical sensor presents some drawbacks. Essentially, because of the reaction involved, it is not an ideal sensor (too long reaction time, exigency of a highly acidic medium prohibiting all in situ measurements, irreversibility of the reaction).

The development of an optical fiber sensor for the nitrite determination might be an interesting alternative, because it could be used to the nitrate ions determination after their preliminary reduction and the fluorimetric methods for the determination of nitrite concentrations have the advantages to need less acidic media and to be less time consuming.

\section{Acknowledgements}

CEA (DTA-LETI-DEIN, Saclay, France) and IFREMER (DITI-DGO-LIO, Centre de Brest, France) are acknowledged for their close collaboration to this work, as well as MENRT for its financial support and INSERM (M.-C. Carré).

\section{References}

1. Greenberg, A. E.; Clesceri, L. S.; Eaton, A. D. In: Standard methods for the examination of water and waste water, 1992, American Public Health Association, Washington, DC, 18th edn.

2. MacCarthy, P.; Klusman, R. W.; Cowling, S. W.; Rice, J. M., Anal. Chem. 1993, 65, 244R.

3. Viriot, M. L.; Mahieuxe, B.; Carré, M. C.; André, J. C.; Analusis 1995, 23, 312.

4. Axelrod, H. D.; Bonelli, J. E.; Lodge Jr. J. P., Anal. Chim. Acta 1970, 51, 21.

5. Miguel, A. H.; Braun, R. D., J. Chem. Educ. 1974, 51, 682.

6. Viriot, M. L.; André, J. C., Analusis 1989, 17, 91.

7. Handbook of Chemistry and Physics, 72th Ed., RD Lide, 1991-1992, 8.

8. Fuh, M. R. S.; Burgess, L. W.; Hirschfeld, T.; Christian, G. D.; Wang, F., Analyst 1987, 112, 1159.

9. Mahieuxe, B.; Carré, M. C.; Viriot, M. L.; André, J. C.; Donner, M., J. Fluoresc. 1994, 4, 7.

10. Gaucher, J. C.; Rougeault, S.; Salles, A.; Seigneur, A., In: Capteurs Bio-Chimiques, Report to the MENRT by the CEADEIN-CE, Saclay LETI-France, 1993.

11. Unger, K. K.; Becker, N.; Roumeliotis, P., J. Chromatogr. 1976, 125, 115.

12. Carré, M. C.; Mahieuxe, B.; Darne-Hosotte, R.; Viriot, M. L.; André, J. C., Entropie 1995, 192-193, 34.

13. Lavorel, J., J. Phys. Chem. 1957, 61, 1600.

14. Hauser, P. C., Tan, S., Analyst 1993, 118, 991. 